\title{
Improving Soil Heat Flux Accuracy with the Philip Correction Technique $\mathscr{a}$
}

\author{
BING TONG \\ Climate and Weather Disasters Collaborative Innovation Center, Jiangsu Key Laboratory of Agricultural Meteorology, \\ College of Applied Meteorology, Nanjing University of Information Science and Technology, Nanjing, China \\ THOMAS J. SAUER \\ USDA-ARS, National Laboratory for Agriculture and the Environment, Ames, Iowa \\ ZHIQIU GAO \\ State Key Laboratory of Atmospheric Boundary Layer Physics and Atmospheric Chemistry, IAP, CAS, Beijing, and \\ College of Atmospheric Physics, Nanjing University of Information Science and Technology, Nanjing, China \\ XINHUA XIAO \\ Biological and Environmental Sciences Department, Alabama A\&M University, Normal, Alabama \\ ROBERT HORTON \\ Agronomy Department, Iowa State University, Ames, Iowa
}

(Manuscript received 21 November 2018, in final form 7 May 2019)

\begin{abstract}
Soil heat flux $G_{s}$ is an important component of the surface energy balance. Soil heat flux plates (SHFPs) are widely used to measure $G_{s}$, although several errors are known to occur. The Philip correction has been applied to minimize errors in $G_{s}$ measured by SHFPs $\left(G_{p}\right)$ if the soil thermal conductivity $\lambda_{s}$, SHFP thermal conductivity $\lambda_{p}$, and plate geometry function $H$ are known. The objective of this study is to evaluate the effectiveness of the Philip correction for a variety of SHFPs. The $\lambda_{p}$ were determined without thermal contact resistance and differed from the manufacturer-specified $\lambda_{p}$. A simplified $H$ formulation was similar to or less than the full $H$ equation for different SHFP shapes. The $G$ ratio $\left(G_{p} / G_{s}\right)$ was sensitive to $\lambda_{s} / \lambda_{p}$ and $H$ when they were relatively small. Compared with the $G_{s}$ determined by a gradient method ( $G_{s}$ grad), the $G_{p}$ measured under a full corn (Zea mays, L.) canopy in the field underestimated $G_{s}$ by $38 \%-62 \%$. After applying the Philip correction, almost all $G_{p}$ agreed better with $G_{s-}$ grad. Generally, the $G_{p}$ corrected with measured plate parameters agreed better with $G_{s \_}$grad than those corrected with manufacturer-specified values. The $G_{p}$ corrected with the simplified and full $H$ expression differed for different SHFPs. These results indicate that SHFPs always underestimate $G_{s}$ and that the performance of the Philip correction is affected by $\lambda_{p}$, plate dimensions, and $H$. An alternative method to measure $G_{s}$ by a three-needle heat-pulse sensor or a gradient method, in which soil temperature and water content are measured at several depths, is recommended.
\end{abstract}

\section{Introduction}

Soil heat flux $G_{s}$, the amount of thermal energy that moves through an area of soil in a unit of time, is a

\footnotetext{
Supplemental information related to this paper is available at the Journals Online website: https://doi.org/10.1175/JHM-D-180243.s1.
}

Corresponding author: Thomas J. Sauer, tom.sauer@usda.gov component of the surface energy balance. In addition to being a component of the surface energy balance, soil heat flux impacts the soil thermal regime. Soil heat flux $G_{s}$ can be measured with the calorimetric, gradient and combination methods (Kimball and Jackson 1979; Fuchs 1986; Sauer 2002; Sauer and Horton 2005), all of which require accurate measurements of soil thermal properties and temperature. Soil heat flux plates (SHFPs) are used to directly measure $G_{s}$ at a shallow depth. SHFPs are often small, rigid, disc-shaped sensors with constant 
thermal properties that are inserted horizontally into the soil to measure $G_{s}$.

However, $G_{s}$ measured by SHFPs $\left(G_{p}\right)$ have potential errors, as first described by Philip (1961). The errors may be caused by (i) liquid water and vapor flow divergence due to the impermeability of the SHFP, (ii) thermal contact resistance between the SHFP and the soil matrix due to poor physical contact at the SHFP-soil interface, and (iii) heat flow divergence near the SHFP due to the thermal conductivity of SHFP $\lambda_{p}$ being different from the $\lambda$ of the surrounding soil $\lambda_{s}$ (Philip 1961; Fuchs and Hadas 1973; Mayocchi and Bristow 1995). All SHFPs are constructed of materials with fixed $\lambda_{p}$ under ambient conditions, while $\lambda_{s}$ varies with soil mineral type, particle size, organic matter, bulk density, and especially water content $\theta$ (de Vries 1963). It is relatively difficult to quantify the first two errors, and they are minimal under some conditions. There is often a diffuse drying front in the surface soil layer, so if the SHFP is buried at a depth $\geq 5 \mathrm{~cm}$, the error due to latent heat loss should not be significant ( $\mathrm{Lu}$ et al. 2016). The thermal contact resistance at the plate-soil interface is expected to decrease with increasing $\theta$ and decreasing particle size (Fuchs and Hadas 1973; Hadas 1974; van Haneghem et al. 1983). However, heat flow divergence always occurs to some degree due to $\lambda_{s}$ differing from $\lambda_{p}$.

Several previous studies have attempted to minimize errors from heat flow divergence. Philip (1961) established an equation for correcting plate fluxes using $\lambda_{s}, \lambda_{p}$, and a plate dimension function $H$ [see Eqs. (1)-(7) in section 2]. The Philip correction is a common method used to correct SHFP $G_{s}$. Mogensen (1970) tested the Philip (1961) method. Sauer et al. (2003) evaluated the Philip correction and concluded that its application generally improved agreement between SHFP estimates and independent $G$ measurements, especially for $\lambda_{p}<$ $\lambda_{s}$. However, most corrected SHFP $G_{p}$ values were still significantly lower than the actual $G_{s}$ values. Using the measured $\lambda_{p}$ value instead of the manufacturer-specified $\lambda_{p}$ value and plate dimensions with a simplified $H$ expression, Ochsner et al. (2006) and Sauer et al. (2007) corrected $G_{p}$ with the Philip equation and the correction improved the consistency but not the average agreement between independently measured $G_{s}$ and corrected SHFP $G_{p}$. Sauer et al. (2008a) also corrected SHFP $G_{p}$ measured in agar by the Philip correction using manufacturer-specified and measured $\lambda_{p}$ and plate dimensions with the simplified $H$ expression. Overall, using the measured SHFP parameters (Sauer et al. 2007) failed to improve agreement between the $G_{p}$ and the agar $G$. Thus, the Philip correction does not always improve SHFP measurements of $G_{s}$.

Sauer et al. (2003) noted that some manufacturers provide a $\lambda_{p}$ value that is the $\lambda$ of the material that comprises the core or majority of the SHFP, while other manufacturers provide a measured $\lambda$ value. No standard method for determining $\lambda_{p}$ exists. Most SHFP calibrations are completed in sandy soil and thermal contact resistance at the plate-soil interface is not considered. Mogensen (1970) reported that there are considerable discrepancies between the simplified and full $H$ expressions. Sauer et al. (2003) examined the simplified and full $H$ expressions and reported that, in their study, the differences were small. To our knowledge, there are no reports on calibrating SHFPs in media without some thermal contact resistance at the SHFP-medium interface. Also, few studies have examined differences between the full $H$ and simplified $H$ expressions, and their influences on the $G$ ratio are not fully known. We hypothesize that (i) the manufacturerspecified $\lambda_{p}$ is not always accurate and (ii) the simplified $H$ expression may lead to relatively large errors, which can impact the accuracy of the correction made to $G_{s}$ measured by SHFPs.

An alternative approach to address heat flow distortion errors is to use a self-calibrating plate, such as the Hukseflux HFP01SC plate (Hukseflux Thermal Sensors, Delft, the Netherlands). The plate has a heater in it to do a self-calibration to change the plate sensitivity based on the soil thermal conductivity. The primary disadvantage of this self-calibrating plate method is that the plate is relatively large. Therefore, it is difficult to install without disturbing the soil, and it also will distort the water content and temperature of the overlying soil (Ochsner et al. 2006; Peng et al. 2015). Other studies have reported that improved sensor technology has allowed the gradient method to become a viable alternative to the heat flux plate method (Cobos and Baker 2003; Ochsner et al. 2006; Heitman et al. 2008a; Sauer et al. 2008b; Peng et al. 2015; Lu et al. 2016).

The overall objective of this study is to evaluate the effectiveness of the Philip correction for SHFPs of widely contrasting designs. The three specific objectives are (i) to calibrate the $\lambda$ of SHFPs in agar-stabilized water, (ii) to examine the differences and influences of the full and simplified $H$ expressions, and (iii) to use field data to determine the effectiveness of the Philip correction on SHFP performance with manufacturer-specified and measured $\lambda_{p}$ and dimensions by comparing with soil heat flux determined by the gradient method.

\section{Materials and methods}

\section{a. Laboratory experiment}

Four commercially available SHFPs with a range of thermal conductivity $\lambda$, thickness $T$, and length $L$ or diameter $D$ were evaluated (Table 1, GHT-1C, Int. 
Thermal Instr. Co., Del Mar, CA; CN3, Carter-Scott Manu. Pty. Ltd., Brunswick, Victoria, Australia; HFT1.1, Radiation and Energy Balance Systems, Seattle, WA; 610, C. W. Thornthwaite Assoc., Pittsgrove, NJ). Three individual sensors of each design of SHFP were used. The SHFPs were placed in a calibration box $(46 \mathrm{~cm} \times 51 \mathrm{~cm} \times$ $8.9 \mathrm{~cm})$ filled with agar-stabilized water $\left(10 \mathrm{~g} \mathrm{~L}^{-1}\right)$ in the laboratory to provide one dimensional heat flow. A heated plate and heat sink were located at the bottom and upper surface of the agar, respectively. Thermocouples (0.254-mm-diameter copper-constantan) were placed in the center of the box $1.5,3,4.5,6$, and $7.5 \mathrm{~cm}$ above the heated plate to measure the temperature profile within the agar. Electrical power at four voltages was applied to the heated plate to provide heat fluxes of $21,43,86$, and $172 \mathrm{~W} \mathrm{~m}^{-2}$ in the calibration box. Calibration runs were completed for several days at constant voltage (flux) until steady-state conditions were achieved at each flux. The SHFP $G$ data were recorded each hour, and $24 \mathrm{~h}$ of data at each flux under steady-state conditions were used for further analysis. The $\lambda$ of the agar $\lambda_{a}$ was $0.567 \pm$ $0.004 \mathrm{~W} \mathrm{~m}^{-1} \mathrm{~K}^{-1}$ (mean \pm one standard deviation), determined using Fourier's law during the plate calibration runs. Additional information about the laboratory experimental setup can be found in Sauer et al. (2008a).

\section{b. Field experiment}

The same plates of the four SHFP designs used in the laboratory study were used in a field study again with three sensors of each design. They were installed in three interrows under a full maize (Zea mays, L.) canopy, near Ames, Iowa, on 25 June 2009 (DOY 176). The soil at the site had a loam texture (25\% clay, $41.2 \%$ silt, and $33.8 \%$ sand). The rows were $76 \mathrm{~cm}$ apart and ran east-west. One plate from each manufacturer was placed in each interrow approximately $25 \mathrm{~cm}$ apart. All the plates were in the middle of the interrow at a depth of $4.5 \mathrm{~cm}$. A profile of type $\mathrm{T}$ (copper-constantan) thermocouples (0.254-mm diameter) were placed in each of the three interrows at depths of $0.9,2.1,4.5,10,25$, and $50 \mathrm{~cm}$. Previous research has reported that the number of soil temperature measurements required for accurate characterization of the temperature profile depends upon the rapidity at which the temperature at the soil surface fluctuates (Horton et al. 1983). In this study, there were more soil temperature measurements close to the soil surface than in the deeper layers and three sets of thermocouples in three different crop interrows to assure that the temperature profile measurements are representative. Three soil moisture sensors (ML2X, Delta-T Devices Ltd., Burwell, Cambridge, United Kingdom) were placed in the outer two interrows at depths of 2,10 , and $25 \mathrm{~cm}$. An infrared thermometer (IRTS, Campbell Scientific, Inc., 
Logan, UT) was positioned $45 \mathrm{~cm}$ above the interrow to measure the soil surface temperature. All of the soil heat flux, temperature and moisture data were measured at a 15-min interval. The below-canopy net radiation $R_{n}$ was measured with a line net radiometer (TRL, Delta-T Devices, Cambridge, United Kingdom) positioned across an adjacent interrow at a 5-min interval.

Soil core samples for water content $\theta$ were collected on 13 July, and soil core samples for bulk density were collected in the 0-3- and 4-7-cm soil layers in the middle row on 10 August (DOY 222) and in the north and south rows on 7 October (DOY 280). Soil texture in the 0-3and 4-7-cm soil layers were measured using the pipette method (Day 1965; Green 1981). In the same field, a weather station measured and recorded rainfall (Model TE525 tipping-bucket precipitation gauge, Texas Electronics), net radiation above the crop canopy (Model CNR-1 net radiometer, Kipp and Zonen), and other weather components (Xiao et al. 2014).

\section{c. The Philip correction}

Philip (1961) applied a solution to Laplace's equation for steady-state heat conduction in an ellipsoid (oblate spheroid) with a known thermal conductivity embedded in an infinite region having a different thermal conductivity (Carslaw and Jaeger 1959, p. 427). Philip (1961) assumed that the oblate spheroid approximated the shape of a heat flux plate and derived a solution,

$$
f=\frac{\varepsilon}{1+(\varepsilon-1) H(\eta)},
$$

where $f$ is termed as the $G$ ratio, $\varepsilon$ is termed as the $\lambda$ ratio, and $H(\eta)$ is the plate geometry function. The $G$ ratio is defined as the ratio of $G_{p}$ to $G_{s}$ such that $f=G_{p} / G_{s}$, where $G_{p}$ and $G_{s}$ are the heat flux through the plate and the soil, respectively. The $\lambda$ ratio $\varepsilon$ has $\varepsilon=\lambda_{p} / \lambda_{s}$, where $\lambda_{p}$ and $\lambda_{s}$ are the thermal conductivity of plate and the surrounding soil or media. The plate geometry function $H(\eta)$ has its full expression as below:

$$
H(\eta)=\frac{1}{1-\eta^{2}}-\frac{\eta}{\left(1-\eta^{2}\right)^{3 / 2}} \tan ^{-1} \frac{\left(1-\eta^{2}\right)^{1 / 2}}{\eta},
$$

where $\eta=b / a$, with $b$ and $a$ being the dimensions of minor and major axis, respectively. By assuming that $\eta$ is "small," Philip simplified the full $H$ function of Eq. (2) as follows:

$$
H(\eta)=1-(\pi / 2) \eta
$$

For a thin square plate with thickness $T$ and side length $L$,

$$
H=1-1.70 \frac{T}{L}
$$

For a thin circular plate of diameter $D$,

$$
H=1-1.92 \frac{T}{D}
$$

Finally, combining Eqs. (1) and (4) gives

$$
f=\frac{1}{1-1.70 \frac{T}{L}\left(1-\varepsilon^{-1}\right)},
$$

and combining Eqs. (1) and (5) gives

$$
f=\frac{1}{1-1.92 \frac{T}{D}\left(1-\varepsilon^{-1}\right)} .
$$

The values of $\alpha$ in Eqs. (6) and (7) (1.70 and 1.92, respectively) were derived by Philip (1961), but they have been the source of some disagreement. Philip (1961) also calculated a square plate $\alpha$ of 1.31 from Portman (1958) data, noting that it was "a somewhat open question which of the values 1.31 or 1.70 is to be preferred" and further that "the discrepancy is perhaps rather trivial" (p. 573). Sauer et al. (2003) reported that use of $\alpha=1.31$ instead of 1.70 as shown in Eq. (6) was found to provide better overall agreement between corrected and known $G$ for the GHT-1C and CN3 plates. In this study, 1.31 was used in Eqs. (4) and (6).

\section{d. The gradient method to estimate soil heat flux}

Soil heat flux at the $4.5-\mathrm{cm}$ depth was estimated by the gradient method ( $G_{s \_}$grad $)$, as follows:

$$
G_{s \_} \operatorname{grad}=-\lambda_{s} \frac{d T}{d z} \text {. }
$$

The soil temperature gradient $d T / d z$ values at each time step were calculated as the differentiation of $T(z)$ at the $z=4.5-\mathrm{cm}$ depth, where $T(z)$ values were fitted by a cubic spline function with the soil temperature $T(z)(z=$ $0.9,2.1,4.5,10,25$, and $50 \mathrm{~cm})$ measured at the six depths. Nassar and Horton (1989) reported that the cubic spline function provided better soil temperature parameters (amplitude and phase angle of the temperature wave) with depth than the second- and third-order Taylor polynomials. The differentiation method can give a more reasonable temperature gradient compared with the linear method $(\Delta T / \Delta z)$ which is often used. Yang and Wang (2008) suggested that the linear method 
always underestimated the soil temperature gradient, thus, underestimating the soil heat flux. The accuracy of thermocouple measurement and the sensor depth change have impact on the soil temperature gradient. For clarity, the uncertainty of the temperature gradient for 2.1- and 10-cm depths is analyzed with the linear method as follows: if the uncertainty of the temperature measurement is $0.1^{\circ} \mathrm{C}$, the temperature gradient uncertainty is $0.2^{\circ} \mathrm{C} / 0.079 \mathrm{~m}=2.53^{\circ} \mathrm{Cm}^{-1}$; if the depth change is $0.01 \mathrm{~cm}$, the temperature gradient uncertainty is $0.2^{\circ} \mathrm{C} /(0.079-0.02) \mathrm{m}=3.39^{\circ} \mathrm{Cm}^{-1}$.

The values of soil temperature gradient in the middle interrow were about half of those in the other two interrows as the temperatures in the middle interrow were less than those in the north and south interrows. The potential reasons for soil temperature disagreements between the middle interrow and the other two interrows are the nonhomogenous soil conditions. No soil water contents were measured in the middle interrow either, therefore, the temperature measurements from the middle interrow were not used and the average measurement values from the north and south interrows were used for further analysis.

The $\lambda_{s}$ values were estimated by the Lu et al. (2014) model, an empirical model for estimating $\lambda$ from soil texture, bulk density, and water content [see Eqs. (3)(6) in their paper for details]. Peng et al. (2015) reported that the $\lambda_{s}$ values estimated by the $\mathrm{Lu}$ et al. (2007) model were similar to the $\lambda_{s}$ values measured by a heat-pulse sensor, with a root-mean-square error (RMSE) $0.02 \mathrm{~W} \mathrm{~m}^{-1} \mathrm{~K}^{-1}$. Lu et al. (2014) reported that the estimation errors of the Lu et al. (2014) model were acceptable and were slightly lower than those of the $\mathrm{Lu}$ et al. (2007) model. The average soil texture and bulk density $\left(1.24 \pm 0.01 \mathrm{~g} \mathrm{~cm}^{-3}\right)$ were measured in the $0-7-\mathrm{cm}$ soil layer at the north and south rows on 7 October, and water content at the $4.5-\mathrm{cm}$ depth $\left(\theta \_4.5 \mathrm{~cm}\right)$ was obtained by linear interpolation using $\theta$ measured at the 2 and $10-\mathrm{cm}$ depths. In this study, only water contents varied with time as the bulk density was considered relatively stable. The sensitivity of $\lambda_{s}$ on dynamic bulk density values are shown in Fig. S4 in the online supplemental material.

\section{e. Statistical analysis}

The RMSE and the normalized RMSE (NRMSE) were calculated to evaluate the difference between the raw $G_{p}$ or the Philip corrected $G_{p}$ and $G_{s \_}$grad,

$$
\mathrm{RMSE}=\sqrt{\frac{\sum_{i=1}^{n}\left(X_{m}-X\right)^{2}}{n}}, \quad \text { and }
$$

$$
\text { NRMSE }=\sqrt{\frac{\sum_{i=1}^{n}\left(X_{m}-X\right)^{2}}{\sum_{i=1}^{n} X^{2}}},
$$

where $n$ is the total number of data points, $X_{m}$ is raw or Philip corrected $G_{p}$, and $X$ is $G_{s \_} \operatorname{grad}$.

\section{Results}

\section{a. $\lambda_{p}$ determined by the Philip correction using agar measurements in the laboratory}

Figure 1 shows that, except for the HFT1.1, all of the other plates significantly underestimated $G$ in agar by an average of $13.5 \%-34.1 \%$. The manufacturer-specified $\lambda_{p}$ of the HFT1.1, $1.0 \mathrm{~W} \mathrm{~m}^{-1} \mathrm{~K}^{-1}$, is larger than the measured $\lambda_{a}, 0.56 \mathrm{~W} \mathrm{~m}^{-1} \mathrm{~K}^{-1}$, causing the plate $G$ to be slightly larger than the agar $G\left(G_{a}\right)$ at the two larger flux runs. The $\lambda_{p}$ of the other three plates are less than $\lambda_{a}$, resulting in underestimates of $G$. Our results were similar to those reported by Sauer et al. (2003) for measurements in sand with the same apparatus. They reported that SHFPs underestimated $G_{s}$ in dry sand by $2.4 \%-38.5 \%$ and by $13.1 \%-73.2 \%$ in saturated sand, while in a moist clay in the field, SHFPs ranged from $6.2 \%$ overestimation to $7.14 \%$ underestimation compared to $G_{s}$ determined by the gradient method.

The measured $G$ of the agar and SHFPs, plate dimensions (Sauer et al. 2007) and $\lambda_{a}$ values, were used to estimate $\lambda_{p}$ from Eq. (1) using the full $H$ expression [Eq. (2)]. The average calibrated $\lambda_{p}$ values in agar are shown in Table 1 with the manufacturer-specified $\lambda_{p}$ and dimensions. The $\lambda_{p}$ values of the SHFPs ranged from 0.163 (measured $\lambda_{p}$ of 610 ) to $1.0 \mathrm{~W} \mathrm{~m}^{-1} \mathrm{~K}^{-1}$ (manufacturer-specified $\lambda_{p}$ of HFT1.1), which varied by a factor of 6.15. Except for the GHT-1C, the measured $\lambda_{p}$ values were less than the manufacturerspecified $\lambda_{p}$ values by $22 \%-51 \%$.

\section{b. The full and simplified $H$ functions and corresponding $G$ ratio}

After determining the $\lambda_{p}$ values of the SHFPs, the plate geometry function $H$ was the only factor impacting the calculation of the $G$ ratio for a given $\lambda_{s}$. Table 1 shows that the length and diameter measured with digital calipers with accuracy of $0.03 \mathrm{~mm}$ (721B, The L.S. Starrett Co., Athol, MA) at four locations on triplicate plates of each design are from $1.9 \%$ less than to $3.9 \%$ greater than the manufacturer-specified dimensions. Thicknesses measured at five locations on the same plates were from $3.1 \%$ less than to $56.4 \%$ greater than the manufacturer-specified values. Dimension ratios 

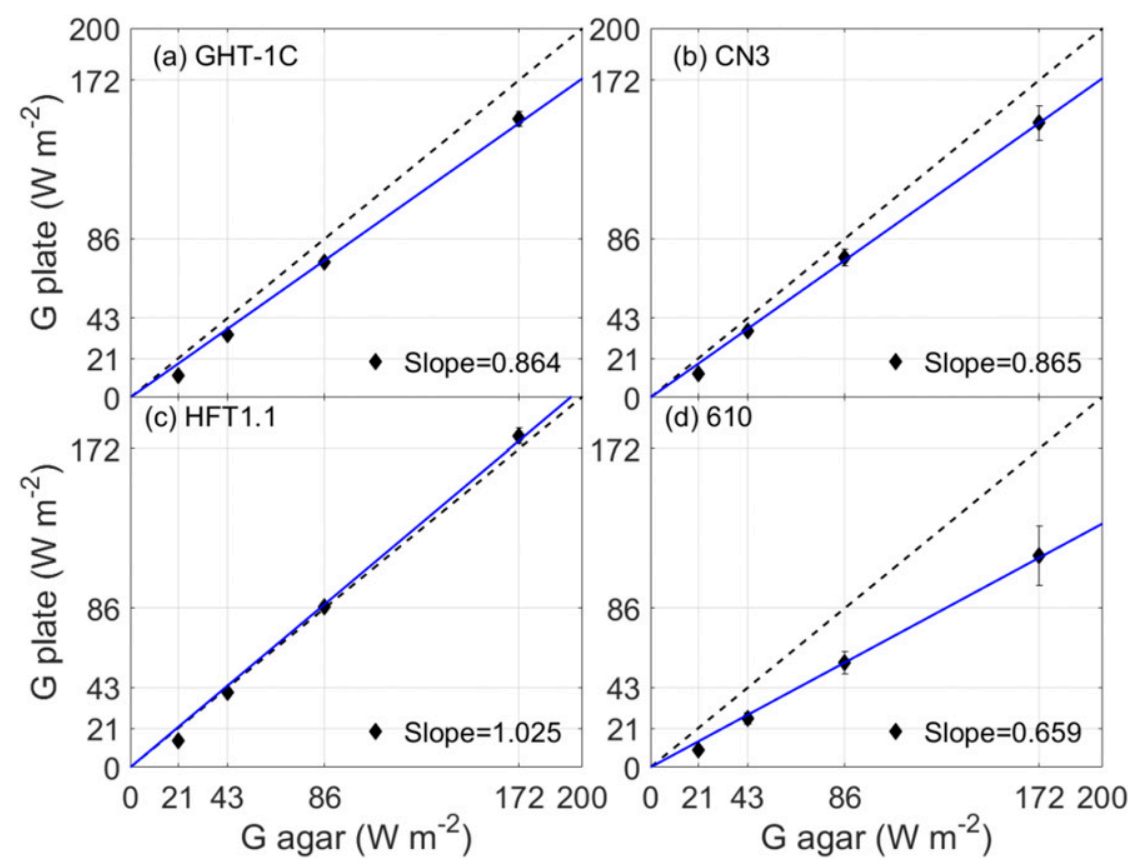

FIG. 1. The average $G$ of agar vs the SHFP $G$ for the (a) GHT-1C, (b) CN3, (c) HFT1.1, and (d) 610 at $21,43,86$, and $172 \mathrm{~W} \mathrm{~m}^{-2}$, respectively. The error bar of each point represents one standard deviation of SHFP $G$ with 24 measurements. The dashed diagonal lines are the $1: 1$ line.

$\eta(T / L$ or $T / D)$ were $24 \%$ less than to $52 \%$ greater than the manufacturer-specified values. The $H$ values calculated by the simplified and full expressions with the manufacturer-specified and measured dimensions of the four SHFPs, respectively, are shown in Fig. 2. The $H$ values decreased with increasing $\eta$, specifically $T / L$ for square plates or $T / D$ for circular plates. The $H$ values ranged from 0.70 (610 with measured parameters and full $H$ ) to 0.88 (GHT-1C with measured parameters and simplified $H$ ), and the differences were within $19 \%$. The simplified $H$ values of the square plates (GHT-1C and CN3) were close to the full $H$ values, while the simplified $H$ values of the circular plates (HFT1.1 and 610) were lower than the full $H$ values, and their differences increased with increasing dimension ratio.

The performance of a given SHFP was a function of $\lambda_{p} / \lambda_{s}$ and $H$. The predicted $G$ ratios for each SHFP design for $\lambda_{s}$ between 0.2 and $2.5 \mathrm{~W} \mathrm{~m}^{-1} \mathrm{~K}^{-1}$ are shown in Fig. 3 . Generally, the errors in estimated $G$ were larger for smaller $\lambda_{p}$ and were as large as $-80 \%(610)$ with simplified $H$ and measured parameters and $-73 \%(610)$ with full $H$ and manufacturer-specified parameters at $\lambda_{s} \gg \lambda_{p}$ for this SHFP. The distinct contrasts in the trend and magnitude of $G$ ratios were due to the combined effects of the different $\lambda_{p}$ (Table 1) and $H$ values (Fig. 2). The $G$ ratios decreased with increasing $\lambda_{s}$ at a given $\lambda_{p}$ and $H$, and decreased with decreasing $\lambda_{p}$ for different SHFPs. The variations in $\lambda_{p}$ were larger than those in $H$, and $\lambda_{p}$ dominated the $G$ ratio trends. In Fig. 3a, the $G$ ratio curves for the SHFPs with $\lambda_{p}=0.69 \mathrm{~W} \mathrm{~m}^{-1} \mathrm{~K}^{-1}$ (HFT1.1) was the largest followed by the SHFPs with $\lambda_{p} \leq 0.31 \mathrm{~W} \mathrm{~m}^{-1} \mathrm{~K}^{-1}(\mathrm{GHT}-1 \mathrm{C}$ and $\mathrm{CN} 3)$, and the smallest was for the SHFP with $\lambda_{p}=0.16 \mathrm{~W} \mathrm{~m}^{-1} \mathrm{~K}^{-1}$ (610). In Fig. 3b, the order of the $G$ ratio curves was HFT1.1 $>$ GHT1.1 $\approx \mathrm{CN} 3 \approx 610$. In Figs. $3 \mathrm{c}$ and $3 \mathrm{~d}$, the order of the $G$ ratio curves was HFT1.1 $>$ GHT1.1 $\approx$ CN3 $>$ 610. In Fig. 3d, the $G$ ratios with full $H$ were less than those with simplified $H$.

In Figs. 3a and 3b, similar $H$ values resulted in similar $G$ ratios, and the larger $H$ values led to $G$ ratios closer to one. The $G$ ratios of the square GHT-1C and CN3 were similar, because the simplified and full $H$ values were similar, while the $G$ ratios of the circular HFT1.1 and 610 with simplified $H$ were much smaller than those with full $H$. All of the $G$ ratio values with simplified and full $H$ expressions were relatively close when the manufacturerspecified parameters were used.

\section{c. Test of the Philip correction for $G_{s}$ values measured by SHFPs in the field}

Field $G_{s}$ data from DOYs 240-263 were used to illustrate the performances of the different SHFPs and examine the performance of the Philip correction under 


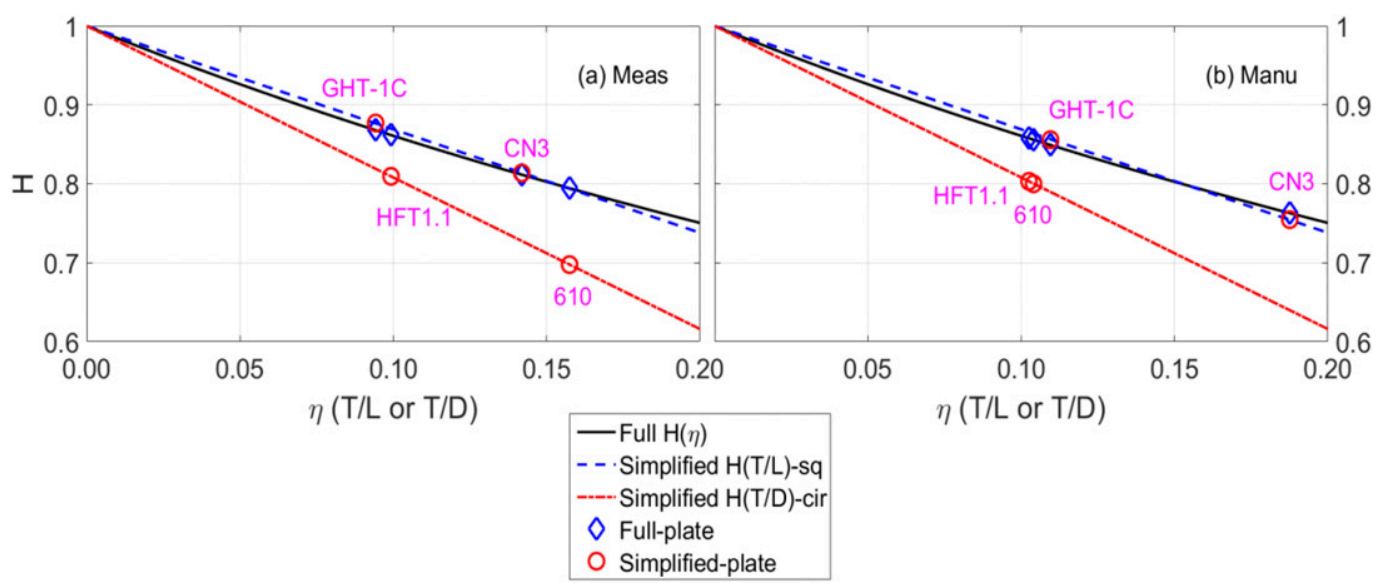

FIG. 2. The shape factor $H$ obtained by the full [Eq. (2)] and simplified [Eqs. (4) and (5)] expressions with (a) measured (meas) and (b) manufacturer-specified (manu) parameters for the GHT-1C, CN3, HFT1.1, and 610. Note the simplified $H$ for square plates (GHT-1C and CN3) was calculated by Eq. (4) with $\alpha=1.31$ instead of $\alpha=1.70$.

field conditions. The two weeks had mostly sunny days with moist soil following $30 \mathrm{~mm}$ of rainfall during DOYs 235-239. The soil temperature gradient, $\lambda_{s}$, and $G_{s \_}$grad values at the $4.5-\mathrm{cm}$ depth for the south interrow were slightly larger than those for the north interrow, with slopes of 1.02, 1.03, and 1.05, respectively, for DOYs 240-263 (Fig. S5). The $\lambda_{s}$ values for the south interrow were larger than those for the north interrow due to the $\theta$ values measured in the south interrow being larger than those in the north (Fig. S3). The average $\lambda_{s}$ estimated by the $\mathrm{Lu}$ et al. (2014) model was $0.99 \pm$ $0.04 \mathrm{~W} \mathrm{~m}^{-1} \mathrm{~K}^{-1}$ at an average $\theta$ of $0.27 \pm 0.02 \mathrm{~m}^{3} \mathrm{~m}^{-3}$. The average $G$ ratios estimated by the Philip equation with full [Eqs. (1) and (2)] and simplified $H$ [Eq. (6) or Eq. (7)] for the manufacturer-specified and the measured parameters are shown in Table 2.

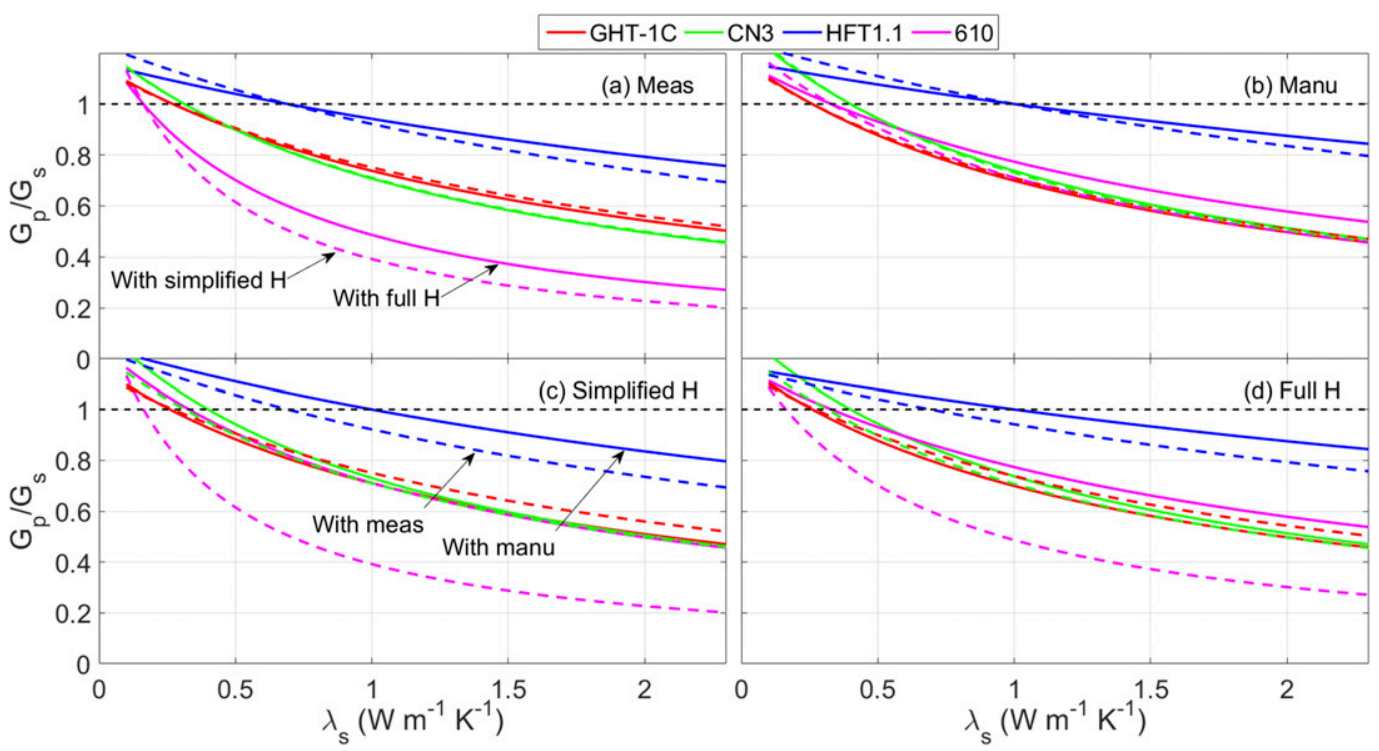

FIG. 3. Predicted $G$ ratios $\left(G_{p} / G_{s}\right)$ for the GHT-1C, CN3, HFT1.1, and 610 at varying $\lambda_{s}$ with the (a) measured (meas) and (b) manufacturer-specified (manu) parameters using simplified [Eq. (6) or (7)] and full $H$ [Eqs. (1) and (2)], and (c) simplified and (d) full $H$ with the measured and manufacturer-specified parameters, respectively. In (a) and (b), the curves with the same color are for one SHFP with the same $\lambda_{s}$ and different $H$ expressions, respectively. In (c) and (d), the curves with the same color have the same $H$ expression with the measured and manufacturer-specified parameters, respectively. 
TABLE 2. The mean values of predicted $G$ ratio of SHFPs estimated by full and simplified $H$ with manufacturer-specified (manu) and measured (meas) parameters, respectively, with standard deviation within 0.015 . The average $\lambda_{s}$ value was $0.99 \pm$ $0.04 \mathrm{~W} \mathrm{~m}^{-1} \mathrm{~K}^{-1}$.

\begin{tabular}{llc}
\hline \hline \multirow{2}{*}{ Plate } & \multicolumn{2}{c}{ By full (simplified) $H$} \\
\cline { 2 - 3 } GHT-1C & Manu & Meas \\
CN3 & $0.70(0.71)$ & $0.74(0.75)$ \\
HFT1.1 & $0.74(0.73)$ & $0.71(0.71)$ \\
610 & $1.00(1.00)$ & $0.94(0.92)$ \\
\hline
\end{tabular}

Almost all of the $G$ ratios were less than one, ranging from 0.40 to 1.00 , which indicated that most of the SHFPs underestimated the actual heat flux under these field conditions. Generally, the lower the $\lambda_{p}$ values, the lower the $G$ ratio values. The 610 had the lowest estimated $G$ ratio, with 0.49 for full and 0.40 for simplified $H$ expressions with the measured parameters. The $G$ ratios of the HFT1.1 were slightly larger than one with the manufacturer-specified parameters and smaller than one (within 0.08) with the measured values. Except for the GHT-1C, the $G$ ratios of the other three SHFPs with measured parameters were smaller than those with manufacturer-specified parameters. The ratios of SHFPs $G_{p}$ and $G_{s-}$ grad did not follow the same trend with $\lambda_{s}$ as shown in Fig. 3 (data not shown). In Fig. 3, $G$ ratios were calculated by the Philip equation with the measured and manufacturer-specified dimensions, $\lambda$ of SHFP and soil and showed the errors of $G_{p}$ measured by SHFPs due to heat flow distortion. Thus, the $G$ ratios only represented some conditions and assumptions. The ratios of measured $G_{p}$ and $G_{s_{-}}$grad represented the actual field conditions and included all the potential errors caused by heat flow distortion, water flow distortion and thermal contact resistance.

The SHFP $G_{p}$ values were corrected as the ratio of $G_{s}$ measured by SHFP (raw $G_{p}$ ) to the predicted $G$ ratio. The raw $G_{p}$ values, corrected with the Philip equation with manufacturer-specified (manu) and measured (meas) parameters were compared to the gradient method $G_{s}$ values ( $G_{s \_}$grad).

Figure 4 shows the results of SHFP $G_{p}$, their corrections with the Philip correction using the full $H$ expression and the $G_{s-}$ grad for typical wet and dry days. Compared with the $G_{s-\text { grad, all of the SHFPs underestimated the } G_{s}}$ amplitude on the dry day, with numerically smaller fluxes during the daytime and nighttime. The 610 had the largest differences with the $G_{s-}$ grad, and the underestimations (overestimations) were up to $44(25) \mathrm{W} \mathrm{m}^{-2}$ during the daytime (nighttime). In the laboratory experiments, the HFT1.1 had the best performance, while its performance was slightly worse than the GHT-1C and CN3 in the field. The exact reasons remain unknown. Possible reasons include the combined impact of plate dimension and thermal conductivity. In the field experiments, the $\lambda_{p}$ of the HFT1.1, GHT-1C, and CN3 were much less than $\lambda_{s}$,
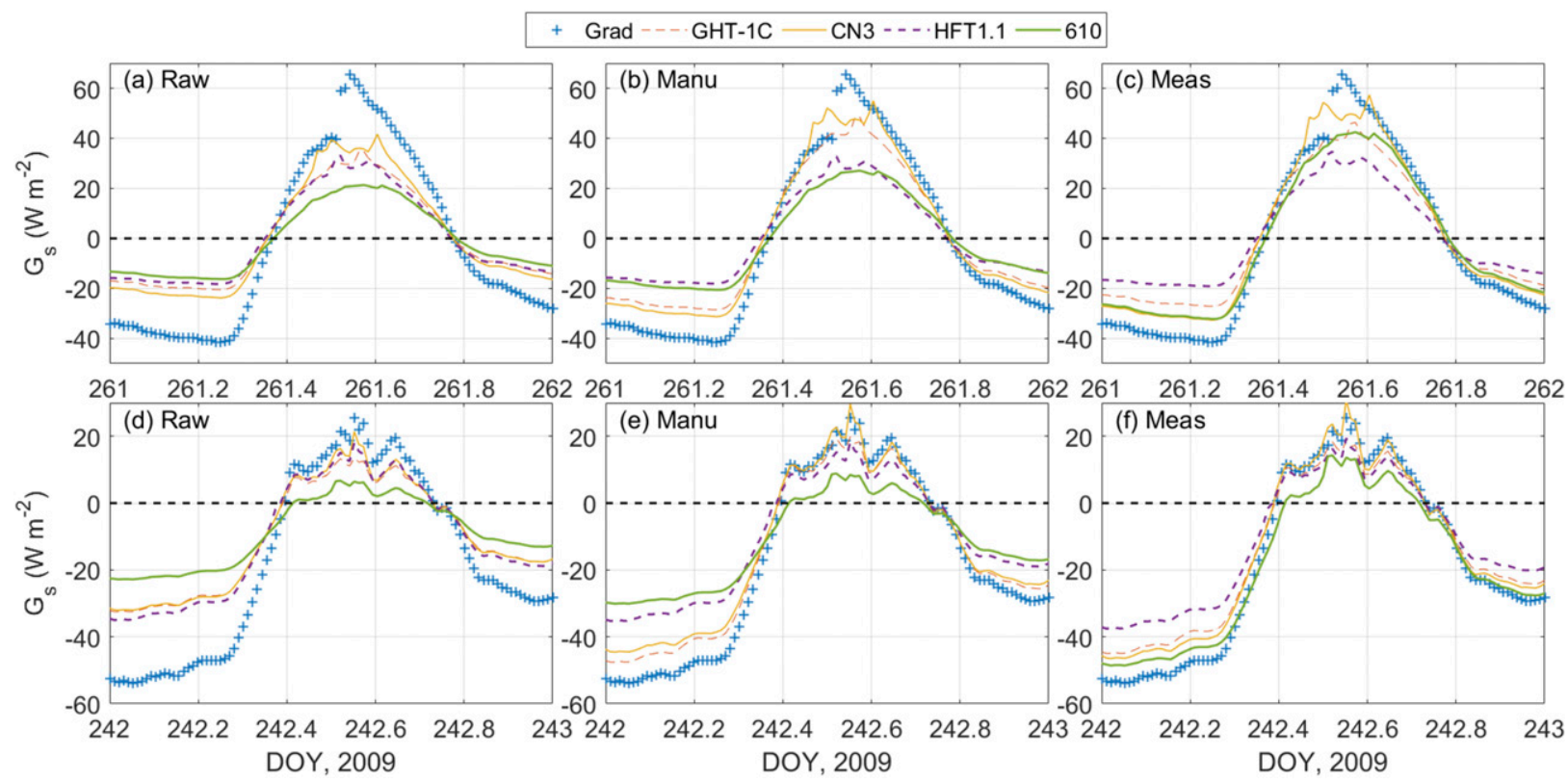

FIG. 4. The variations of $G_{s}$ measured by SHFPs (Raw), corrected by the Philip correction with manufacturer-specified (manu) and measured (meas) parameters with full $H$, and estimated by the gradient method (grad) at the 4.5-cm depth for two typical days, (a)-(c) a typical dry day (DOY 261) and (d)-(f) a typical wet day (DOY 242). 
resulting in the $G_{p}$ of the HFT1.1, GHT-1C, and CN3 being quite similar, even though the $\lambda_{p}$ of the HFT1.1 was larger than the other two SHFPs. It is also notable that the GHT-1C and CN3 both have metal exteriors, which seem to provide better performance under field conditions.

Note that there is a jump of about $20 \mathrm{~W} \mathrm{~m}^{-2}$ of $G_{s-} \operatorname{grad}$ around noon on DOY 261. Figure S1(a) shows that the below-canopy $R_{n}$ fluctuated around noon and increased sharply from about $110 \mathrm{~W} \mathrm{~m}^{-2}$ at 1200 local time (LT) to $160 \mathrm{~W} \mathrm{~m}^{-2}$ at 1205 and $1210 \mathrm{LT}$ on DOY 261. The surface temperatures responded to the change in net radiation. The soil temperatures measured in the south interrow on DOY 261 at 1215 (1230) LT were about $1.3^{\circ} \mathrm{C}\left(1.2^{\circ} \mathrm{C}\right)$ greater than the temperature at 1200 (1215) LT at the $0.9-\mathrm{cm}$ depth and $0.6^{\circ} \mathrm{C}\left(1.3^{\circ} \mathrm{C}\right)$ greater at the $2.1 \mathrm{~cm}$ depth, while for the deeper depths, the temperature differences were relatively stable and less than $0.3^{\circ} \mathrm{C}$ (Figs. S1 and S2). The surface soil temperature jump caused the soil temperature profile changes and a sharp increase in temperature gradient at the $4.5-\mathrm{cm}$ depth (Fig. S2). Thus, the $G_{s-}$ grad increased sharply. A

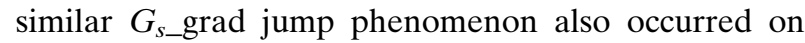
several other days. The potential reason was that, when solar radiation reached the soil surface under the full maize canopy intermittently, especially near solar noon, the topsoil was heated quickly but the deeper soil was not, which caused a temperature gradient jump and soil heat flux jump. It seems that the $G_{p}$ did not present a similar jump as the $G_{s \_}$grad did. The potential reasons were that the small increase in the soil surface temperature did not propagate to the deeper depth, and the SHFP was not sensitive to the relatively small temperature change. Ochsner et al. (2006) reported that soil water content and temperature were disrupted by the disks, and temperature difference across the plate plastic disks (below minus above) buried $2 \mathrm{~cm}$ beneath a bare soil surface ranged from $1^{\circ}$ to $3.5^{\circ} \mathrm{C}$.

After making a correction with the manufacturerspecified parameters, the HFT1.1 and 610 still greatly underestimated the amplitude of $G_{s \_}$grad, while the other two SHFPs agreed much better with the $G_{s \_}$grad. Only the HFT1.1 still much underestimated the $G_{s-}$ grad values after correction with measured parameters. For the wet day, $G_{s}$ values were relatively small during the daytime. All of the SHFPs except for the 610 underestimated the $G_{s-}$ grad magnitudes during the nighttime but agreed well with the $G_{s-}$ grad during the daytime. After the Philip correction, all the SHFP $G_{p}$ values were much closer to the $G_{s-}$ grad values, except for the HFT1.1 and 610 with manufacturerspecified parameters and HFT1.1 with measured parameters, respectively. The $G_{p}$ values corrected with the simplified $H$ expression had a similar pattern with small differences in magnitude (data not shown).
Because the soil had $\lambda_{s}$ values 0.4-5.1 times greater than the measured SHFPs $\lambda_{p}$ values, the magnitudes of $G_{p}$ values for all the SHFPs were expected to be significantly smaller than those of $G_{s}$ values. The data were in agreement with this expectation. For the entire period the $G_{p}$ underestimated the $G_{s-}$ grad, and after the correction with the full $H$ expression, all of the $G_{p}$ agreed better with the $G_{s_{-}}$grad to varying degrees (Fig. 5). The results of SHFPs $G_{p}$ values corrected by the simplified $H$ expression were similar to those corrected by the full $H$ expression (data not shown). Note that the differences between the average values of $G_{p} / G_{s-} \operatorname{grad}$ in the north and south interrows were $0.02,0.14,0.17$, and -0.04 for the four SHFPs, respectively, and the potential reason of the differences is the nonhomogenous soil conditions.

The values of slope, RMSE, and normalized RMSE of SHFP $G_{p}$ and their correction with the full and simplified $H$ expressions are shown in Fig. 6. The raw $G_{p}$ underestimated $G_{s-}$ grad from around $40 \%$ (GHT-1C, CN3, and HFT1.1) to $62 \%(610)$. The slopes of SHFPs $G_{p}$ corrected with measured parameters were larger than those with manufacturer-specified values with the exception of the GHT-1, and the slopes with full and simplified $H$ expressions were similar with the exception of the 610 (the slope difference between with full $H$ and measured parameters and with simplified $H$ and measured parameters was about 0.20 ). Even though the absolute RMSE values were less than $20 \mathrm{~W} \mathrm{~m}^{-2}$, the relative RMSE values reached up to $62 \%$ (610). No matter whether the plate values were corrected with the full or simplified $H$ expressions, the slopes of the HFT1.1 increased slightly from 0.57 up to 0.62 . The RMSE (NRMSE) values of the corrected GHT-1C and CN3 were within $9 \mathrm{~W} \mathrm{~m}^{-2}(25 \%)$. The 610 corrected with simplified $H$ and measured parameters had the largest slope of $98 \%$, due to its simplified $H$ being underestimated the most (Fig. 2), thus its $G$ ratio was underestimated the most and its correction was overestimated the most. After correction with manufacturer-specified parameters, the RMSE (NRMSE) values of the HFT1.1 slightly increased due to its $\lambda_{p}$ being overestimated and slightly larger than the $\lambda_{s}$.

In a bare field study, Ochsner et al. (2006) reported that the raw $G_{p}$ underestimated $G_{s}$ from $23 \%$ (HFT1.1) to $66.3 \%$ (610) compared to the $G_{s-} \mathrm{grad}$ measured by a three-needle heat pulse sensor. For corn and soybean sites, Ochsner et al. (2006) reported that the HFT1.1 $G_{p}$ values underestimated the $G_{s \_} \operatorname{grad}$ values by $26.3 \%$ and $17.8 \%$, respectively. Similar to the present study, Ochsner et al. (2006) reported that the $G_{p}$ of the 610 improved the most (32.4\%) after the Philip correction with the simplified $H$ expression and 

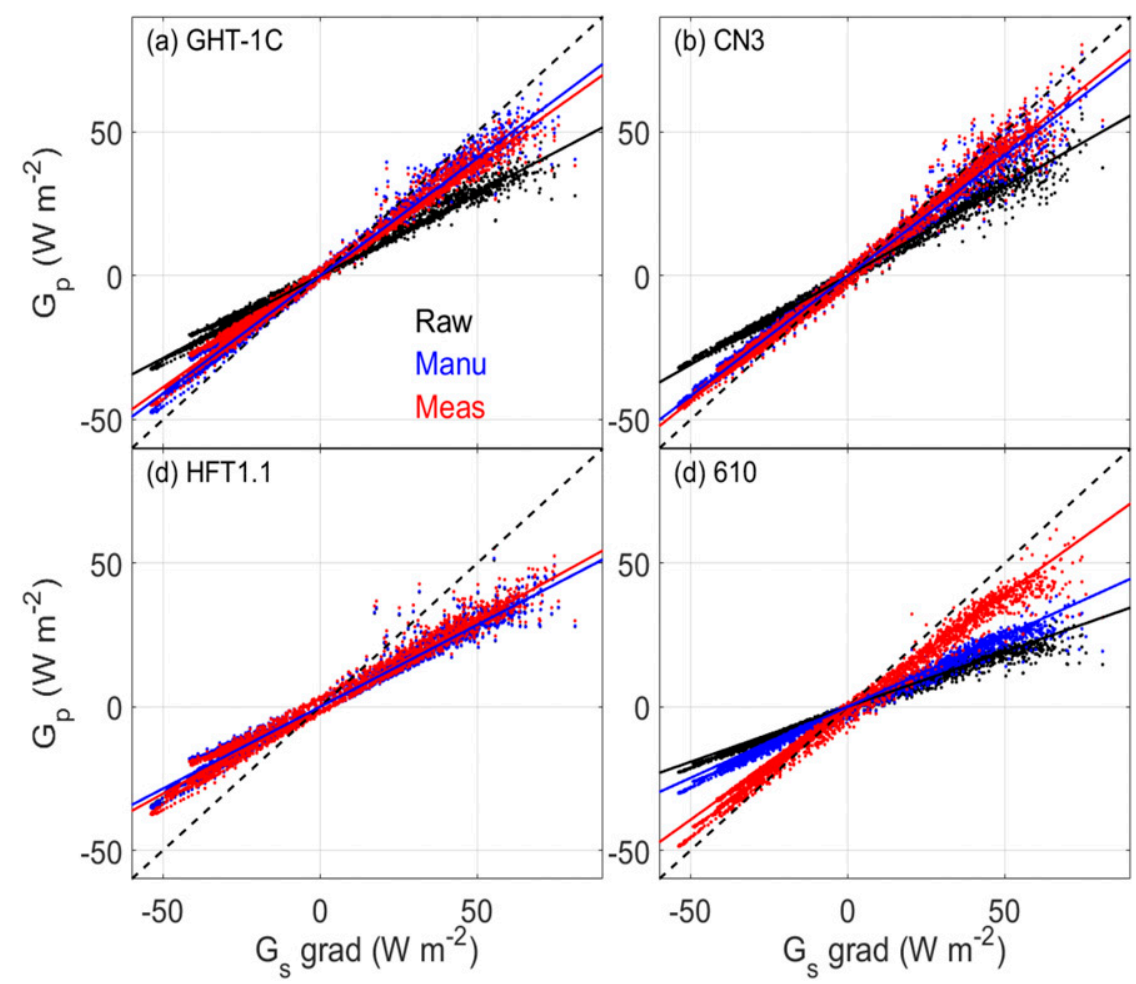

FIG. 5. $G_{p}$ [raw $G_{p}$, and $G_{p}$ corrected with measured (meas) and manufacturer-specified (manu) parameters by full $H$ expression] against $G_{s_{-}}$grad.

manufacturer-specified parameters, while the Philip correction improved the $G_{p}$ of the other SHFPs with changes of $-1.4 \%$ (HFT1.1 at the soybean site) to $13.2 \%$ (CN3 at the bare soil site). The differing Philip correction results obtained in this study compared to the Ochsner et al. (2006) study were caused by the different values used for SHFP $\lambda_{p}$, dimensions, and $H$ expression.

\section{Discussion}

\section{a. Typical calibration method and the present calibration method using agar}

Most previous research on SHFP performance included laboratory determined calibrations obtained in soil with $\lambda_{p}$ values measured using Eq. (8). The results were inevitably influenced by thermal contact resistance between the SHFP and the soil, which was expected to increase with decreasing $\theta$ and increasing particle size (Fuchs and Hadas 1973; Hadas 1974; van Haneghem et al. 1983). For the same types of SHFPs used in this study, Sauer et al. (2007) reported calibrated $\lambda_{p}$ values in quartz sand (GHT-1C, CN3, HFT1.1, and 610 were $0.60,0.63$, 1.26 , and $0.21 \mathrm{~W} \mathrm{~m}^{-1} \mathrm{~K}^{-1}$, respectively), which were from $36 \%$ less (610) to $142 \%$ greater (GHT-1C) than the manufacturer-specified values. The $\lambda_{p}$ reported by Sauer et al. (2007) were from $30 \%$ (610) to $121 \%$ (GHT1C) greater than the measured values in this study. During the calibration, thermocouples were cemented with adhesive to the top and bottom of each SHFP. Temperature gradients across the exterior of the SHFPs were small and prone to error if there was poor contact between the plate and the thermocouple(s). The combination of poor contact between plate and soil and incorrect temperature gradient led to uncertainty in the measured $\lambda_{p}$ values when calibrating in soil. Another potential source of error with this approach was that all of the SHFPs were composed of multiple materials with different $\lambda$ values. The GHT-1C and CN3 were clad with metal but contained plastic or composite materials inside. All of the SHFPs also included some area around their thermopile core that acted as a "guard," so that heat flow through the center of the plate where the thermopile was located was distorted less than at the edges of the sensor. These nonuniformities of $\lambda_{p}$ could contribute to errors in application of the Philip correction that assumes a constant and uniform $\lambda_{p}$.

The present study utilized plate calibrations in agar that provided excellent thermal contact between plate and media, effectively eliminating errors due to thermal contact resistance. 

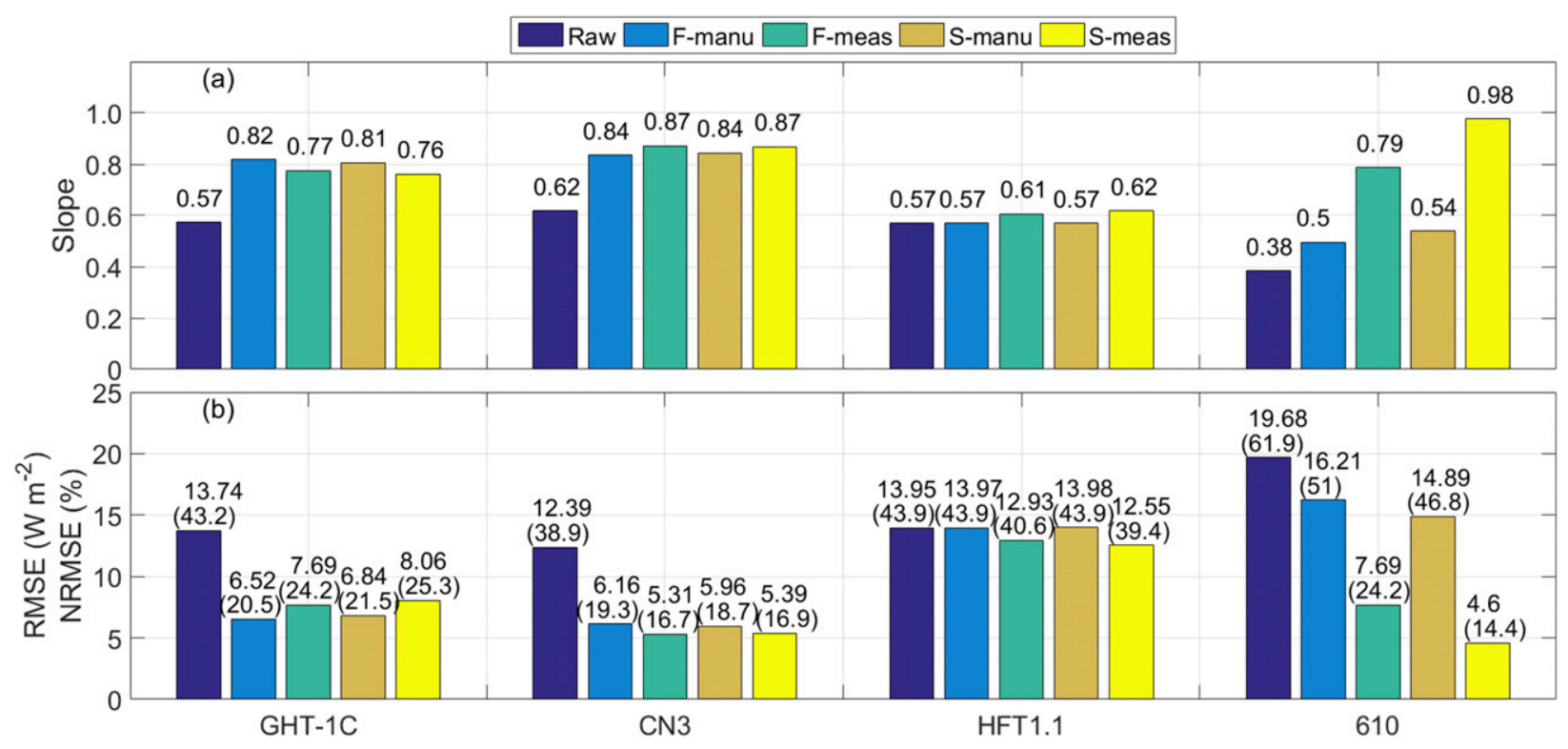

FIG. 6. Linear regression statistics and mean absolute (relative) differences for $G_{s}$ values measured by SHFPs (raw), corrected with manufacturer-specified (manu) and measured (meas) parameters with full and simplified $H$ expressions vs $G_{s}$ values estimated by the gradient method. The F-manu, F-meas, S-manu, and S-meas denote the results of SHFPs by the full (F) and simplified (S) $H$ with manufacturer-specified and measured parameters, respectively. All the $R^{2}$ values are larger than 0.98 .

\section{b. The uncertainty of $\lambda_{p}$ and of plate dimensions}

We assumed no thermal contact resistance and water flow divergence occurred in the agar, leaving only heat flow divergence as a source of error due to the different $\lambda$ values of agar and the SHFPs. The agar-measured $\lambda_{p}$ values were determined by the $G$ of agar and SHFPs, $\lambda_{a}$, and plate dimensions. The $G$ of agar was known, $\lambda_{a}$ was determined using the known $G_{s}$ and temperature gradient in the agar, and plate dimensions were reported by Sauer et al. (2007). The remaining possible error was from $G_{p}$. Uncorrected plate $G_{p}$ values based on the manufacturer calibration factor were used to determine the $\lambda_{p}$ values. Accuracy of the measured $\lambda_{p}$ values was thus dependent on the accuracy of the original manufacturer calibration factor. Sauer et al. (2007) concluded that use of the plate $G_{p}$ values to determine $\lambda_{p}$ was reasonable.

The $G_{p} / G_{a}$ values of SHFPs tended to be most consistent at the higher fluxes of 86 and $172 \mathrm{~W} \mathrm{~m}^{-2}$. Therefore, only data from the two larger flux runs were used to determine $\lambda_{p}$ from the Philip equation. The standard deviation of HFT1.1 $\lambda_{p}$ was about $0.09 \mathrm{~W} \mathrm{~m}^{-1} \mathrm{~K}^{-1}$, and the other three SHFPs were within $0.02 \mathrm{~W} \mathrm{~m}^{-1} \mathrm{~K}^{-1}$. The reason that the HFT1.1 had a relatively large standard deviation was unknown. The poor results of the Philip correction for the HFT1.1 may have been caused by a relatively large uncertainty of $\lambda_{p}$. The slopes of the GHT-1C, CN3, and HFT1.1 were similar (Fig. 5), thus we felt that they had similar $\lambda_{p}$. Therefore, the measured $\lambda_{p}$ of the HFT1.1 may have been overestimated as well as the $G$ ratio of the HFT1.1, causing the Philip correction for the HFT1.1 to be incorrect.

Note that in Table 1 the 610 thickness was underestimated by $50 \%$ by the manufacturer and the thermal conductivity overestimated by a similar amount. Perhaps the underestimated thickness contributed to the overestimated thermal conductivity. For a given SHFP, if the thickness $T$ is underestimated (overestimated), the $\lambda$ ratio $\varepsilon$ is overestimated (underestimated), namely, $\lambda_{p}$ is overestimated (underestimated) based on the Eqs. (6) and (7).

\section{c. Full $H$ and simplified $H$ expressions}

Few studies investigated the differences due to the full $H$ and simplified $H$ expressions. The Philip equation assumed that the oblate spheroid was either a square or a circle with rounded edges, while actual SHFPs are flat with relatively sharp edges. The simplified form of $H$ described in Eq. (3) also assumed that $\eta$ was "small"; however, no criteria were given.

With the manufacturer-specified parameters of SHFPs, Sauer et al. (2003) reported that a comparison of the full and the simplified $H$ expressions introduced a small but systematic error when the approximation was used (their Fig. 5). The errors ranged from $2.9 \%$ for the HFP01SC to $10.6 \%$ for the $\mathrm{CN} 3$ and could effectively increase the magnitude of the $G_{p}$ corrections by these percentages. They concluded that use of the full $H$ expression did not significantly improve the performance of the correction. 


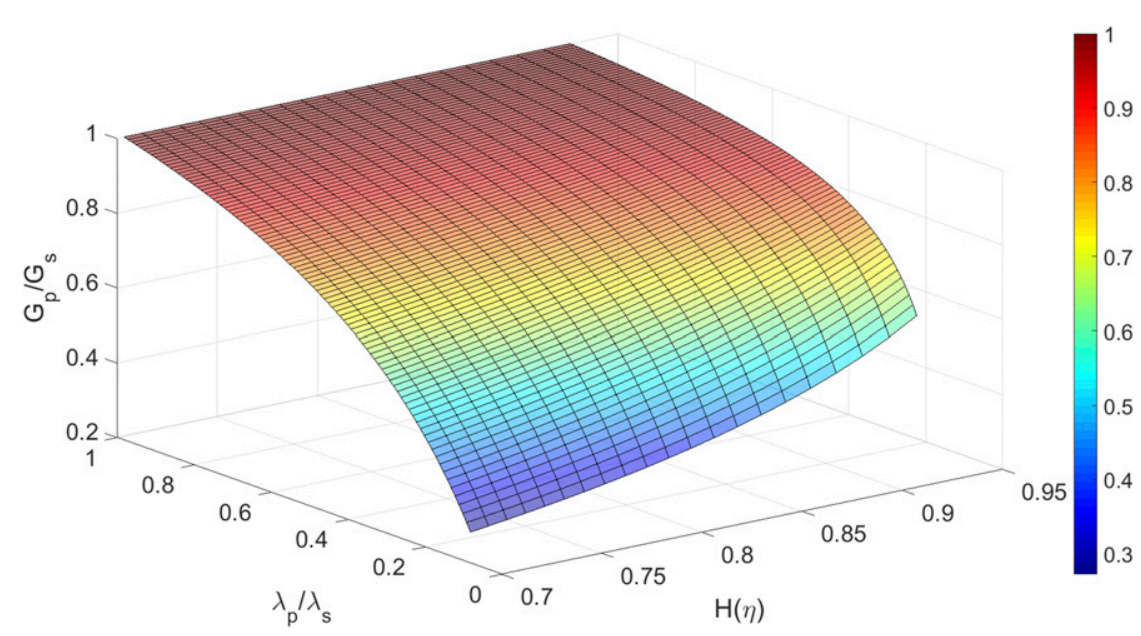

FIG. 7. The $G$ ratio $\left(G_{p} / G_{s}\right)$ vs $\lambda_{p} / \lambda_{s}$ vs $H(\eta)$.

Mogensen (1970), however, reported that there were considerable discrepancies $(13 \%)$ between the simplified $H$ and the full $H$.

The significant discrepancies between manufacturerspecified and actual plate dimensions were unexpected. Our results indicated that the differences between the full and simplified $H$ values were related to the $H$ expression and plate dimensions. The full $H$ value was always larger than the simplified $H$ value for circular SHFPs, and the difference increased with increasing dimension ratio. Based on these results, use of the full $H$ expression is recommended.

\section{d. G ratio as a function of $\lambda_{p} / \lambda_{s}$ and $H$}

Figure 7 shows that the $G$ ratio is sensitive to $\lambda_{p} / \lambda_{s}$ and $H$ when both are relatively small. When one of the two variables $\lambda_{p} / \lambda_{s}$ and $\mathrm{H}$ is held constant, the $G$ ratio decreases when the other variable decreases. When $H$ decreases, $T / L$ or $T / D$ increase, namely, when the plate is thick and/or large. Philip (1961) reported that the thicker the plate the larger the distortion of heat flow. Thus, $G_{p}$ of thicker plate has relatively large errors ( $G$ ratio is small). When $\lambda_{p} / \lambda_{s}$ decreases, the heat flow divergence increases, and $G_{p}$ decreases ( $G$ ratio is small).

For the HFT1.1, the differences between the full and simplified $H$ values were relatively large (Fig. 2), while its $\lambda_{p}$ (Table 1) was the largest, that is, the $\lambda_{p} / \lambda_{s}$ was the largest. Therefore, the $G$ ratio was not very sensitive to the different $H$ values of the HFT1.1. The differences between the manufacturer-specified and the measured dimensions and $\lambda_{p}$ of the 610 were the largest, thus, the differences of $G$ ratio were largest.

The predicted $G$ ratios with measured parameters (Table 2) were closer to the real $G$ ratios than those with manufacturer-specified parameters, except for the GHT-1C (Fig. 6, the slopes of raw $G_{p}$ ). The predicted $G$ ratios $(0.92-1.00)$ for the HFT1.1 were largest and larger than the real $G$ ratios $(0.57)$ by $62 \%-76 \%$.

These results indicated that accurate $\lambda_{p}$, plate dimensions, and $H$ expression all affected the ability of the Philip correction to improve $G_{s}$ measured by SHFPs.

\section{e. $G_{p}$ improvements after correcting for heat flow divergence}

Three potential errors can lead to underestimates of $G_{s}$ when measured by SHFPs. In the present study, SHFPs were buried under a full crop canopy in moist soil of a loamy texture for more than two months before their data were used. The thermal contact resistance was expected to be small after a long period that included several wetting and drying cycles. The net radiation under the full crop canopy was not very large and minimal water and vapor flow divergence was expected. Previous researchers reported that bare soil water evaporation measured by three-needle heat-pulse sensors occurred in the 0-3-cm soil layer (Heitman et al. 2008a,b; Xiao et al. 2011). Mayocchi and Bristow (1995) indicated that if a SHFP was buried too close to the surface (above the drying front), the $G_{s}$ would be underestimated and dense vegetation would reduce the depth to which the drying front will penetrate. Heat flow divergence could contribute significant errors to SHFPs measurements under such conditions. Therefore, the $G_{p}$ corrected by the Philip correction were able to improve $G_{p}$ and reference $G_{s}$ agreement, although different degrees of disagreement still exist. Sauer et al. (2003) indicated that the inconsistent performances of the Philip correction may be due to limitations of the theory, inability to accurately represent flux plate properties, and failure to include other 
factors such as thermal contact resistance and liquid water and vapor flow divergence. Since thermal contact resistance is a function of air gaps between the plate and soil, differences in soil particle size, structure, and water content would all affect contact resistance and thus plate performance. For these reasons, Fuchs and Hadas (1973) and Högström (1974) advocated in situ calibration to assess plate performance and minimize the potentially confounding effects of varying contact resistance. Selfcalibration soil heat flux plates are able to run in situ calibration and can provide relative reasonable $G_{s}$ for some conditions (Ochsner et al. 2006; Peng et al. 2015; Lu et al. 2016), however, the plate interrupts soil water flow due to its relatively large size. Peng et al. (2015) reported that its voltage signals during and shortly after selfcalibration should be discarded from data analysis as the self-heating could bias the voltage signals. The threeneedle heat-pulse sensor (Ren et al. 1999) has the ability to measured $\lambda_{s}$ in situ and is a good candidate to obtain independent soil heat flux using the gradient method (Cobos and Baker 2003; Ochsner et al. 2006; Heitman et al. 2008a,b; Xiao et al. 2011; Peng et al. 2015; Lu et al. 2016; He et al. 2018).

\section{Conclusions}

Although the Philip correction is commonly applied to $G_{s}$ values measured by SHFPs, the correction method provides inconsistent results. Two potential reasons for the inconsistency are that the manufacturer-specified $\lambda_{p}$ is not accurate and use of the simplified $H$ expression may lead to relatively large errors. In this study the $\lambda_{p}$ of the GHT-1C, CN3, HFT1.1, and 610 were measured in agar without thermal contact resistance and water flow divergence, and the measured $\lambda_{p}$ values were generally $22 \%-51 \%$ smaller than the manufacturer-specified $\lambda_{p}$ values. The measured and manufacturer-specified dimensions also differed. Simplified $H$ values for a square plate were similar to the full $H$ values, while for circular plates, the differences were much larger and increased with increasing dimension ratio. The performance of the Philip correction was tested using field data. Field measured SHFPs $G_{p}$ values with the same four designs of SHFPs used in the laboratory test underestimated the $G_{s}$ determined by the gradient method ( $G_{S \_}$grad) by $38 \%$ up to $62 \%$. After the Philip correction, the $G_{p}$ agreed better with $G_{s \_}$grad with different degrees. Generally, the Philip correction with the measured plate characteristics performed better than those with the manufacturer-specified values, and the corrected $G_{p}$ with full $H$ expression were not always close to those with simplified $H$ expression. These results indicated that SHFPs almost always underestimate $G_{s}$, and the performance of the Philip correction is sensitive to $\lambda_{p}$, plate dimensions, and $H$ expression. Careful calibration, accurate plate properties, and use of the full $H$ expression are likely to improve the performance of the Philip correction to limitedly improve the $G_{p}$.

We recommend using $G_{s}$ measured by the threeneedle heat-pulse sensor as a reference to calibrate SHFPs in the field with multiple drying and wetting cycles or measuring $G_{s}$ by the three-needle heat-pulse sensor directly. If a heat-pulse sensor is not available, a gradient method in which soil temperatures and water content are measured at several depths, and an empirical model is used to translate the dynamic soil moisture data into estimate of thermal conductivity, is recommended.

Acknowledgments. We thank the editors and the anonymous reviewers for helpful comments in improving this paper. This work is supported by the National Science Foundation under Grant 1623806, Army Research Office under Grant W911NF-16-1-0287, USDANIFA, Multi-State Project 3188, Iowa State University Department of Agronomy, the Hatch Act, State of Iowa funds, State Scholarship Fund from China Scholarship Council (201608320197), and Jiangsu Province graduate education innovation project (KYLX16_0943).

\section{REFERENCES}

Carslaw, H. S., and J. C. Jaeger, 1959: Conduction of Heat in Solids. 2nd ed. Clarendon Press, 427 pp.

Cobos, D. R., and J. M. Baker, 2003: In situ measurement of soil heat flux with the gradient method. Vadose Zone J., 2, 589-594, https://doi.org/10.2136/vzj2003.5890.

Day, P. R., 1965: Particle fractionation and particle-size analysis. Methods of Soil Analysis: Part 1, C.A. Black et al., Ed., Agron. Monogr., No. 9, Amer. Soc. of Agronomy, 545-567, https:// doi.org/10.2134/agronmonogr9.1.c43.

de Vries, D. A., 1963: Thermal properties of soils. Physics of Plant Environment, W. R. van Wijk, Ed., North-Holland Publications, 210-235.

Fuchs, M., 1986: Heat flux. Methods of Soil Analysis: Part 1Physical and Mineralogical Methods, 2nd ed. A. Klute, Ed., SSSA Book Ser. 5.1, Soil Science Society of America, 957-968, https://doi.org/10.2136/sssabookser5.1.2ed.c40.

_ , and A. Hadas, 1973: Analysis and performance of an improved soil heat flux transducer. Soil Sci. Soc. Amer. J., 37, 173-175, https://doi.org/10.2136/sssaj1973.03615995003700020009x.

Green, A. J., 1981: Particle-size analysis. Manual on Soil Sampling and Methods of Analysis, J. A. McKeague, Ed., Canadian Society of Soil Science, 4-29.

Hadas, A., 1974: Problems involved in measuring the soil thermal conductivity and diffusivity in a moist soil. Agric. For. Meteor., 13, 105-113, https://doi.org/10.1016/0002-1571(74)90068-5.

He, H., M. F. Dyck, R. Horton, T. Ren, K. L. Bristow, J. Lv, and B. Si, 2018: Development and application of the heat pulse method for soil physical measurements. Rev. Geophys., 56, 567-620, https://doi.org/10.1029/2017RG000584. 
Heitman, J. L., R. Horton, T. J. Sauer, and T. M. DeSutter, 2008a: Sensible heat observations reveal soil water evaporation dynamics. J. Hydrometeor., 9, 165-171, https://doi.org/10.1175/ 2007JHM963.1.

— X. Xiao, R. Horton, and T. J. Sauer, 2008b: Sensible heat measurements indicating depth and magnitude of subsurface soil water evaporation. Water Resour. Res., 44, W00D05, https://doi.org/10.1029/2008WR006961.

Högström, U., 1974: In situ calibration of ground heat flux plates. Agric. Meteor., 13, 161-168, https://doi.org/10.1016/00021571(74)90043-0.

Horton, R., P. J. Wierenga, and D. R. Nielsen, 1983: Evaluation of methods for determination apparent thermal diffusivity of soil near the surface. Soil. Sci. Soc. Amer. J., 47, 23-32, https:// doi.org/10.2136/sssaj1983.03615995004700010005x.

Kimball, B. A., and R. D. Jackson, 1979: Soil heat flux. Modification of the Aerial Environment of Plants, B. J. Barfield and J. F. Gerber, Ed., ASAE Monograph, No. 2. ASAE, 211-229.

Lu, S., T. Ren, Y. Gong, and R. Horton, 2007: An improved model for predicting soil thermal conductivity from water content at room temperature. Soil. Sci. Soc. Amer. J., 71, 8-14, https:// doi.org/10.2136/sssaj2006.0041.

- C. Ma, P. Meng, J. Zhang, X. Zhang, Y. Lu, and C. Yin, 2016: Experimental investigation of subsurface soil water evaporation on soil heat flux plate measurement. Appl. Therm. Eng., 93, 433-437, https://doi.org/10.1016/j.applthermaleng.2015.09.058.

Lu, Y., S. Lu, R. Horton, and T. Ren, 2014: An empirical model for estimating soil thermal conductivity from texture, water content, and bulk density. Soil. Sci. Soc. Amer. J., 78, 18591868, https://doi.org/10.2136/sssaj2014.05.0218.

Mayocchi, C. L., and K. L. Bristow, 1995: Soil surface heat flux: Some general questions and comments on measurements. Agric. For. Meteor., 75, 43-50, https://doi.org/10.1016/01681923(94)02198-S.

Mogensen, V. O., 1970: The calibration factor of heat flux meters in relation to the thermal conductivity of the surrounding medium. Agric. For. Meteor., 7, 401-410, https://doi.org/10.1016/ 0002-1571(70)90035-X.

Nassar, I. N., and R. Horton, 1989: Determination of the apparent thermal diffusivity of a nonuniform soil. Soil Sci., 147, 238-244, https://doi.org/10.1097/00010694-198904000-00002.

Ochsner, T. E., T. J. Sauer, and R. Horton, 2006: Field tests of the soil heat flux plate method and some alternatives. Agron. $J .$, 98, 1005-1014, https://doi.org/10.2134/agronj2005.0249.

Peng, X., J. Heitman, R. Horton, and T. Ren, 2015: Field evaluation and improvement of the plate method for measuring soil heat flux density. Agric. For. Meteor., 214-215, 341-349, https://doi.org/10.1016/j.agrformet.2015.09.001.

Philip, J. R., 1961: The theory of heat flux meters. J. Geophys. Res., 66, 571-579, https://doi.org/10.1029/JZ066i002p00571.
Portman, D. J., 1958: Conductivity and length relationships in heat flow transducer performance. Trans. Amer. Geophys. Union, 39, 1089-1094, https://doi.org/10.1029/TR039i006p01089.

Ren, T., K. Noborio, and R. Horton, 1999: Measuring soil water content, electrical conductivity, and thermal properties with a thermo-time domain reflectometry probe. Soil. Sci. Soc. Amer. J., 63, 450-457, https://doi.org/ 10.2136/sssaj1999.03615995006300030005x.

Sauer, T. J., 2002: Heat flux density. Methods of soil analysis: Part 4-Physical Methods, G. C. Topp and J. H. Dane, Ed., SSSA Book Series, No. 5, SSSA, 1233-1248.

— , and R. Horton, 2005: Soil heat flux. Micrometeorology in Agricultural Systems, J. L. Hatfield and J. M. Baker, Eds., ASA Monograph, No. 47, American Society of Agronomy, 131-154, https://doi.org/10.2134/agronmonogr47.c7.

_ D. W. Meek, T. E. Ochsner, A. R. Harris, and R. Horton, 2003: Errors in heat flux measurement by flux plates of contrasting design and thermal conductivity. Vadose Zone J., 2 , 580-588, https://doi.org/10.2136/vzj2003.5800.

_ Heat flow distortion and thermal contact resistance. Agron. J., 99, 304-310, https://doi.org/10.2134/agronj2005.0038s.

— - _ J. L. Heitman, R. Horton, B. D. Tanner, and O. D. Akinyemi, G. Hernandez-Ramirez and T. B. Moorman, 2008a: Careful measurements and energy balance closure - The case of soil heat flux. 28th Conf. on Agricultural and Forest Meteorology, Orlando, FL, Amer. Meteor. Soc., 1.3, https://ams.confex.com/ ams/28Hurricanes/webprogram/Paper138960.html.

—, O. D. Akinyemi, P. Thery, J. L. Heitman, T. M. DeSutter, and R. Horton, 2008b: Evaluation of a new, perforated heat flux plate design. Int. Commun. Heat Mass., 35, 800-804, https:// doi.org/10.1016/j.icheatmasstransfer.2008.03.012.

van Haneghem, I. A., J. Schenk, and H. P. A. Boshoven, 1983: An improved nonsteady-state probe method for measurements in granular materials: II. Experimental results. High Temp. High Press., 15, 367-374.

Xiao, X., R. Horton, T. J. Sauer, J. L. Heitman, and T. Ren, 2011: Cumulative evaporation as a function of depth and time in a developing soil water evaporation zone. Vadose Zone J., 10, 1016-1022, https://doi.org/10.2136/vzj2010.0070.

_ J. L. Heitman, T. J. Sauer, T. Ren, and R. Horton, 2014: Sensible heat balance measurements of soil water evaporation beneath a maize canopy. Soil. Sci. Soc. Amer. J., 78, 361-368, https://doi.org/10.2136/sssaj2013.08.0371.

Yang, K., and J. M. Wang, 2008: A temperature predictioncorrection method for estimating surface soil heat flux from soil temperature and moisture data. Sci. China Ser. D, 51, 721-729, https://doi.org/10.1007/s11430-008-0036-1. 